\title{
AC 2007-678: CREATIVE DESIGN CLASS WITH PATENTS
}

Junghwan Kim, Yonsei University

Il Moon, Yonsei University 


\title{
Creative Design Class with Patents
}

\begin{abstract}
All forty-two students in my class applied for the patents. In Creative Design Class, students have learned that how to generate creative idea and apply for patents with creative inventions. All students understood their type of preference through MBTI (Myers-Briggs Type Indicator) test. They also got their grades of creativity through TTCT (Torrance Tests of Creative Thinking) test. The students have learned about safety engineering, product design and optimization which to develop their creative inventions. All students of this class applied for patents successfully.
\end{abstract}

Forty-two students were divided into 10 groups and each groups applied for a patent with a creative invention. (One group applied for an additional patent.) Eleven inventions were developed in this class; a Solar Powered Hover Craft, Solar Powered Balloon Toy to Propose Her, an Auto Watering Machine with Timer, a Bicycle with built-in a Fuel Cell Powered Charger, a Portable Capsule Shampoo, a Reversely Holding Umbrella, a Solar Powered Airship, a Nude Coffee Mix Bar (You can see the contents of coffee.), a Clothes Rack with Drying, a Ruler with a Sliding Pen and a Spot Removal Machine.

In this class, they had brain storming about designs and the concepts of products and made a schedule to do for about three months. Their ideas of designs, concepts and the real products were presented in the class. The teamwork and communication skills are very important to develop their creative inventions. At the last step of this class, all students participated in Creative Design Contest held in YONSEI University.

\section{Introduction}

What is creativity? There are many definitions of creativity. In the dictionary, the word 'creative' is defined as 'an ability to invent and develop original ideas, especially in the arts.' Creativity has been focused on every field. The lecturer used many methods to improve this creativity in the classroom by keep practicing brainstorming, brain writing, checklist, morphological analysis, etc. These ways of practicing solved the problem either directly or indirectly. Thus, practical experiences have been applied to make the problems to be solved, and make new ideas on the other hand.

Forty-two students in the Creative Design Class have applied for their own patents with creative inventions. Students have taken from elementary steps as followings:

- Myers-Briggs Type Indicator (MBTI) \& Torrance Tests of Creative Thinking (TTCT)

- Lectures

- Creative Invention

- Applying for patents 


\section{Curriculum of Creative Design Class}

The goal of this Creative Design Class is to apply for patents with creative inventions. All the students should follow the curriculum and schedule exactly. There are four steps in this class. Figure 1 shows diagram of curriculum in Creative Design Class.

First, all students are required to understood their type of characteristics through MBTI (MyersBriggs Type Indicator) test. They should be also able to know their grade of creativity through TTCT (Torrance Tests of Creative Thinking) test.

The second step is called knowledge expansion in improvement of creativity. In this step, students need to study lectures related with improvement of creativity such as engineering design, technical administration, patent, product design, optimization and TRIZ.

The goal of third step is to make products with creative invention. In this step, students should make products with creative invention. They need a brain storming about design and concept of the products. Their ideas of designs, concepts and real products are presented in the class. The teamwork and communication skills are the main points to develop their creative inventions.

The goal of last step is to apply for patents. A patent is an official right given only to a person or company and allow making or selling a new product for a certain period of time. Everyone applies for a patent but its procedures are not simple.

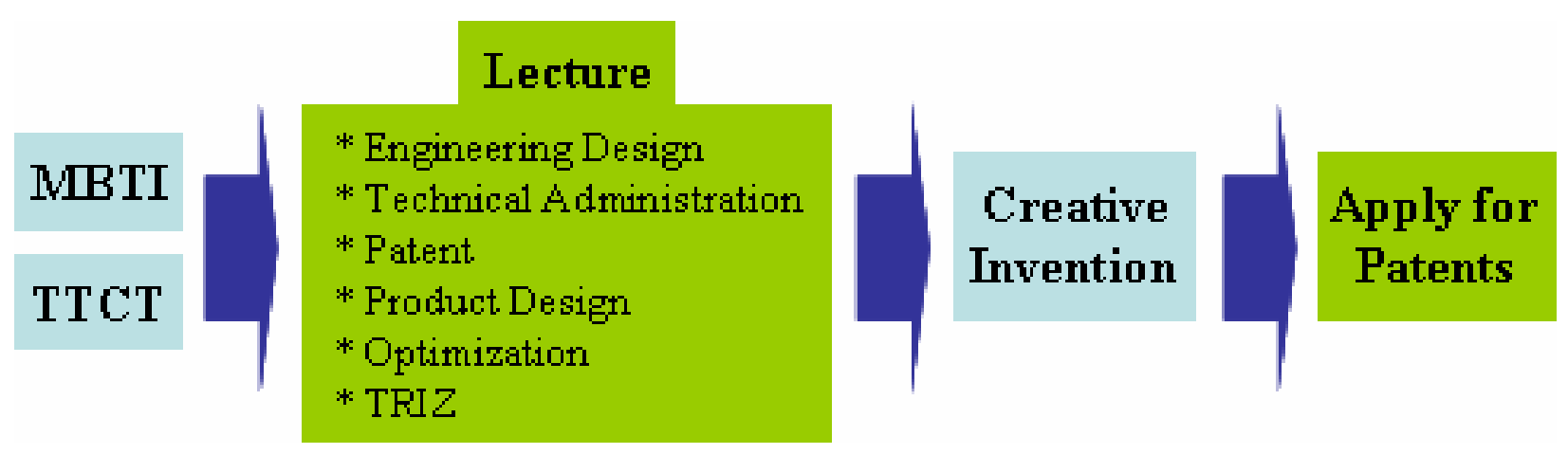

Figure1. Diagram of Curriculum in Creative Design Class

2.1. Myers-Briggs Type Indicator (MBTI) and Torrance Tests of Creative Thinking (TTCT) test

(TTCT) test Myers-Briggs Type Indicator (MBTI) is a personality test designed to assist a person in identifying some significant personal preferences. The types the MBTI sorts for, known as dichotomies are extraversion / introversion, sensing / intuition, thinking / feeling and judging / perceiving. Participants are given one of 16 four-letter abbreviations, such as ESTJ or INFP, indicating what their preferences are. The term best-fit types refers to the ethical code that facilitators are required to follow. It states that the person taking the indicator is always the best 
judge of what their preferences are and that the indicator alone should never be used to make this decision. ${ }^{1}$

All students have had a MBTI test. Table 1 shows the results of MBTI test. The averages of MBTI test in this class are as follows:

- Extraversion(+) or Introversion(-) : +3.571

- Sensing(+) or Intuition(-) : +7.452

- Thinking (+) or Feeling(-) : +13.405

- Judging(+) or Perceiving(-) : -5.548

According to this average, students of this class totally had extraverted attitude. They also got sensing, thinking and judging position.

\begin{tabular}{|c|c|c|c|c|c|c|c|c|c|c|c|c|c|c|c|c|c|c|c|}
\hline \multirow[t]{2}{*}{1} & I & $\mathrm{N}$ & $\mathrm{F}$ & $P$ & \multirow[t]{2}{*}{12} & I & $\mathrm{S}$ & $\mathrm{T}$ & $\mathrm{J}$ & \multirow[t]{2}{*}{23} & $\mathrm{E}$ & S & $\mathrm{T}$ & $\mathrm{J}$ & \multirow[t]{2}{*}{33} & I & $S$ & F & $P$ \\
\hline & 25 & 5 & 15 & 40 & & 39 & 31 & 19 & 1 & & 15 & 19 & 7 & 19 & & 15 & 9 & 13 & 17 \\
\hline \multirow[t]{2}{*}{2} & $\mathrm{E}$ & $S$ & $\mathrm{~T}$ & $P$ & \multirow{2}{*}{13} & $\mathrm{E}$ & $S$ & $\mathrm{~T}$ & $P$ & \multirow[t]{2}{*}{24} & $\mathrm{E}$ & $\mathrm{N}$ & $\mathrm{T}$ & $P$ & \multirow[t]{2}{*}{34} & $\mathrm{E}$ & $\mathrm{N}$ & $\mathrm{T}$ & $P$ \\
\hline & 23 & 14 & 10 & 20 & & 24 & 18 & 15 & 21 & & 31 & 11 & 19 & 55 & & 19 & 9 & 23 & 15 \\
\hline \multirow[t]{2}{*}{3} & $\mathrm{E}$ & $S$ & $\mathrm{~T}$ & $\mathrm{P}$ & \multirow[t]{2}{*}{14} & $\mathrm{E}$ & $S$ & $\mathrm{~T}$ & $\mathrm{P}$ & \multirow[t]{2}{*}{25} & $\mathrm{E}$ & $S$ & $\mathrm{~F}$ & $P$ & \multirow[t]{2}{*}{35} & I & $S$ & $\mathrm{~T}$ & $\mathrm{~J}$ \\
\hline & 27 & 16 & 8 & 4 & & 27 & 10 & 7 & 23 & & 27 & 5 & 17 & 1 & & 47 & 25 & 39 & 39 \\
\hline \multirow[t]{2}{*}{4} & I & $\mathrm{N}$ & $\mathrm{T}$ & $P$ & \multirow[t]{2}{*}{15} & $\mathrm{E}$ & S & $\mathrm{T}$ & $\mathrm{J}$ & \multirow[t]{2}{*}{26} & $\mathrm{E}$ & $\mathrm{N}$ & $\mathrm{F}$ & $\mathrm{P}$ & \multirow[t]{2}{*}{36} & $\mathrm{E}$ & $\mathrm{N}$ & $\mathrm{F}$ & $\mathrm{J}$ \\
\hline & 47 & 3 & 23 & 35 & & 27 & 25 & 7 & 7 & & 1 & 11 & 15 & 39 & & 5 & 3 & 19 & 9 \\
\hline \multirow[t]{2}{*}{5} & $E$ & $S$ & $\mathrm{~T}$ & $\mathrm{P}$ & \multirow[t]{2}{*}{16} & $\mathrm{E}$ & $\mathrm{S}$ & $\mathrm{F}$ & $\mathrm{P}$ & \multirow[t]{2}{*}{27} & I & $S$ & $\mathrm{~T}$ & $\mathrm{~J}$ & \multirow[t]{2}{*}{37} & I & $S$ & $\mathrm{~T}$ & $\mathrm{P}$ \\
\hline & 33 & 5 & 5 & 51 & & 33 & 7 & 21 & 13 & & 25 & 17 & 27 & 3 & & 19 & 21 & 43 & 25 \\
\hline \multirow[t]{2}{*}{6} & $\mathrm{E}$ & $S$ & $\mathrm{~F}$ & $P$ & \multirow[t]{2}{*}{17} & $\mathrm{E}$ & $\mathrm{N}$ & $\mathrm{T}$ & $\mathrm{P}$ & \multirow[t]{2}{*}{28} & $\mathrm{E}$ & $S$ & $\mathrm{~T}$ & $\mathrm{~J}$ & \multirow[t]{2}{*}{38} & I & $S$ & $\mathrm{~T}$ & $\mathrm{P}$ \\
\hline & 19 & 27 & 7 & 11 & & 49 & 3 & 55 & 9 & & 19 & 15 & 30 & 22 & & 17 & 7 & 57 & 3 \\
\hline \multirow[t]{2}{*}{7} & I & $S$ & $\mathrm{~T}$ & $\mathrm{P}$ & \multirow[t]{2}{*}{18} & I & $\mathrm{N}$ & $\mathrm{T}$ & $\mathrm{J}$ & \multirow[t]{2}{*}{29} & I & $\mathrm{N}$ & $\mathrm{T}$ & $\mathrm{J}$ & \multirow[t]{2}{*}{39} & $\mathrm{E}$ & S & $\mathrm{T}$ & $P$ \\
\hline & 27 & 1 & 45 & 17 & & 15 & 7 & 37 & 25 & & 23 & 5 & 9 & 53 & & 23 & 13 & 15 & -18 \\
\hline 8 & $\mathrm{E}$ & $S$ & $\mathrm{~F}$ & $\mathrm{~J}$ & 19 & $\mathrm{E}$ & $S$ & $\mathrm{~F}$ & $\mathrm{P}$ & 30 & I & $S$ & $\mathrm{~T}$ & $\mathrm{~J}$ & 40 & $\mathrm{E}$ & $S$ & $\mathrm{~T}$ & $\mathrm{~J}$ \\
\hline & 24 & 21 & 27 & 21 & & 1 & 19 & 11 & 57 & & 4 & 41 & 7 & 51 & & 23 & 27 & 35 & 45 \\
\hline 9 & $\mathrm{E}$ & $\mathrm{S}$ & $\mathrm{T}$ & P & 20 & $\mathrm{E}$ & $\mathrm{S}$ & $\mathrm{T}$ & P & 31 & $\mathrm{E}$ & $S$ & $\mathrm{~T}$ & $\mathrm{P}$ & 41 & I & $\mathrm{N}$ & $\mathrm{T}$ & $\mathrm{P}$ \\
\hline & 39 & 5 & 23 & 25 & & 29 & 3 & 17 & 1 & & 31 & 3 & 33 & 51 & & 31 & 13 & 27 & 5 \\
\hline 10 & I & $\mathrm{N}$ & $\mathrm{T}$ & $\mathrm{J}$ & 21 & $E$ & $\mathrm{~S}$ & $\mathrm{~T}$ & $P$ & 32 & I & $\mathrm{N}$ & $\mathrm{F}$ & $P$ & 42 & I & $\mathrm{N}$ & $\mathrm{F}$ & $\mathrm{P}$ \\
\hline & 22 & 20 & 17 & 41 & & 13 & 19 & 21 & 17 & & 5 & 5 & 15 & 47 & & 29 & 3 & 3 & 35 \\
\hline 11 & $\mathrm{E}$ & $S$ & $\mathrm{~T}$ & $\mathrm{P}$ & 22 & I & $\mathrm{N}$ & $\mathrm{T}$ & $\mathrm{P}$ & & & & & & & & & & \\
\hline & 5 & 3 & 19 & 9 & & 35 & 15 & 27 & 5 & & & & & & & & & & \\
\hline
\end{tabular}

Table1. Results of MBTI test 
Developed by Dr E. Paul Torrance, the TTCT is a highly reliable and one of the most widely used creativity testing of its kind. This is a test where multiple methods of creative thinking are assessed, recognized and potentially nurtured. These tests come in two forms: Figural and Verbal Students of this class had a TTCT-Figural test. TTCT-Figural is appropriate at all levels, kindergarten through adult. Testing Time is $\mathbf{4 5}$ minutes. It uses three picture-based exercises to assess five mental characteristics ${ }^{2}$ :

- Fluency

- Originality

- Abstractness of titles

- Elaboration

- Resistance to premature closure

Table 2 shows the result of TTCT test. They got their grade of creativity through TTCT (Torrance Tests of Creative Thinking) test.

\begin{tabular}{|c|c|c|c|c|c|c|c|c|c|c|c|c|c|}
\hline$\#$ & Fluency & Originality & $\begin{array}{l}\text { Abstractness } \\
\text { of titles }\end{array}$ & Elaboration & $\begin{array}{l}\text { Resistance to } \\
\text { premature closure }\end{array}$ & AVG & $\#$ & Flvency & Originality & $\begin{array}{c}\text { Abstractness } \\
\text { of titles }\end{array}$ & Elaboration & $\begin{array}{l}\text { Resistance to } \\
\text { premature closure }\end{array}$ & AVG \\
\hline 1 & 99 & 112 & 110 & 140 & 95 & 111.2 & 22 & 83 & 91 & 70 & 140 & 60 & 88.8 \\
\hline 2 & 79 & 83 & 40 & 106 & 60 & 73.6 & 23 & 93 & 101 & 61 & 106 & 51 & 82.4 \\
\hline 3 & 112 & 125 & 92 & 133 & 81 & 108.6 & 24 & 120 & 119 & 40 & 133 & 69 & 96.2 \\
\hline 4 & 117 & 130 & 40 & 133 & 75 & 99 & 25 & 141 & 130 & 81 & 115 & 69 & 107.2 \\
\hline 5 & 83 & 91 & 81 & 106 & 64 & 85 & 26 & 104 & 119 & 156 & 115 & 91 & 117 \\
\hline 6 & 117 & 130 & 61 & 140 & 69 & 103.4 & 27 & 99 & 98 & 127 & 140 & 81 & 109 \\
\hline 7 & 104 & 119 & 86 & 125 & 60 & 98.8 & 28 & 108 & 94 & 70 & 95 & 54 & 84.2 \\
\hline 8 & 96 & 108 & 76 & 125 & 60 & 93 & 29 & 108 & 94 & 92 & 125 & 81 & 100 \\
\hline 9 & 115 & 127 & 86 & 140 & 60 & 105.6 & 30 & 70 & 91 & 92 & 106 & 64 & 84.6 \\
\hline 10 & 90 & 98 & 86 & 158 & 54 & 97.2 & 31 & 79 & 125 & 101 & 115 & 75 & 99 \\
\hline 11 & 87 & 94 & 40 & 95 & 54 & 74 & 32 & 117 & 112 & 76 & 160 & 75 & 108 \\
\hline 12 & 87 & 94 & 81 & 125 & 69 & 91.2 & 33 & 104 & 105 & 122 & 140 & 81 & 110.4 \\
\hline 13 & 139 & 150 & 86 & 133 & 86 & 118.8 & 34 & 120 & 116 & 110 & 106 & 69 & 104.2 \\
\hline 14 & 79 & 83 & 96 & 95 & 75 & 85.6 & 35 & 104 & 119 & 141 & 156 & 95 & 123 \\
\hline 15 & 120 & 133 & 61 & 153 & 60 & 105.4 & 36 & 150 & 150 & 86 & 133 & 86 & 121 \\
\hline 16 & 127 & 141 & 110 & 140 & 81 & 119.8 & 37 & 130 & 116 & 40 & 140 & 75 & 100.2 \\
\hline 17 & 115 & 127 & 81 & 125 & 60 & 101.6 & 38 & 95 & 112 & 76 & 115 & 81 & 95.8 \\
\hline 18 & 80 & 87 & 76 & 125 & 51 & 83.8 & 39 & 96 & 79 & 154 & 115 & 69 & 102.6 \\
\hline 19 & 112 & 125 & 76 & 125 & 60 & 99.6 & 40 & 96 & 98 & 53 & 85 & 95 & 85.4 \\
\hline 20 & 59 & 62 & 53 & 61 & 40 & 55 & 41 & 81 & 66 & 75 & 55 & 55 & 66.4 \\
\hline 21 & 83 & 91 & 81 & 83 & 40 & 75.6 & 42 & 96 & 98 & 53 & 115 & 54 & 83.2 \\
\hline
\end{tabular}

Table2. Results of TTCT test 


\subsection{Lectures}

Before students made creative products, they should study lectures related improvement in creativity. The lectures are:

- Engineering Design

- Technical Administration

- Patent

- Product Design

- Optimization

- TRIZ

All forty-two students had a presentation in their topics, and Table 3 shows the list of presentation in this class.

After a presentation, they asked questions. The presenter answered the question and they exchanged opinions each other. Through this step, they could know both own and other topic.

\begin{tabular}{|c|c|c|c|c|c|}
\hline$\#$ & Field & Subject & \# & Field & Subject \\
\hline 1 & E.D. & Problem Definition & 22 & P.D. & Six Sigma in Product Design and Manufacturing \\
\hline 2 & E.D. & AnIdea Creation & 23 & P.D. & CVD of polysilicon in IC (integrated circuit) manufacture \\
\hline 3 & E.D. & Creative Valuation & 24 & P.D. & Solar desalination unit \\
\hline 4 & E.D. & Idea Decision & 25 & P.D. & What is Product Design \\
\hline 5 & E.D. & Pugh Method & 26 & P.D. & Examples of Product Development Processes (Rapid, Research Intensive) \\
\hline 6 & E.D. & Solution Scheme fulfillment & 27 & P.D. & Examples of Product Development Processes (Systems Industrial Design) \\
\hline 7 & E.D. & What is a Design? & 28 & P.D. & Benchmarking \& Establishing Engineering Specifications \\
\hline 8 & E.D. & Engineering Design Process & 29 & P.D. & Automotive fuel cell \\
\hline 9 & E.D. & Project Plan & 30 & P.D. & Environmental safe refrigerants \\
\hline 10 & E.D. & Decision based on Economical Efficiency & 31 & P.D. & Hem dialysis device \\
\hline 11 & E.D. & Design Document Preparation & 32 & P.D. & Hand warmer \\
\hline 12 & E.D. & Innovation of a Workshop & 33 & Opt. & Quantity of Alcohol which is the most suitable \\
\hline 13 & T.A. & A New Technology development strateg & 34 & Opt. & The Optimum Studying \\
\hline 14 & T.A. & $R \& D$ project manage ment & 35 & Opt. & Matching Problem \\
\hline 15 & T.A. & TRIZ for R\&BD & 36 & Opt. & Casanova \\
\hline 16 & T.A. & R\&BD Leadership & 37 & Opt. & The Dinner of West Hill Family \\
\hline 17 & T.A. & Technical Writing & 38 & Opt. & Terran \\
\hline 18 & Patent & Patent Case1 & 39 & Opt. & Horse Racing \\
\hline 19 & Patent & Patent Case2 & 40 & Opt. & Putting in a Bag \\
\hline 20 & Patent & Patent Case3 & 41 & Opt. & A Popularity Secret of Professor MOON \\
\hline 21 & P.D. & Examples of Product Development Processes & 42 & Opt. & Example \\
\hline
\end{tabular}

Table3. List of Presentation (E.D.; Engineering Design, T.A.; Technical Administration, P.D.; Product Design, Opt.; Optimization) 


\subsection{Creative Invention}

At this step, students are divided into 10 groups. Each group made products with a creative invention.

Students should make products with creative invention. To do so, first of all, they had a brain storming about design and concept of the products and made a schedule to do for three months. Their ideas of design, concept and real products are presented in the class. The teamwork and communication skills are very important to develop their creative inventions.

\subsection{Applying for patents}

All students of this class have applied for patents with creative products. Everyone can apply for a patent but its procedure is not simple. There are lots of required documents and procedures ${ }^{3}$ :

- Required documents:

- Transmittal letter

- Specification

- Claim

- Abstract

- Drawing

- Declaration and Power of Attorney

- Assignment

- Priority documents

- Procedures:

- Primary Amendments

- First Office Action

- Final Office Action

- Notice of Allowance

- Pay Registration Fee \& Submit Formal Drawing

- Letter Patent

All students of this class applied for patents with creative inventions successfully. Table 4 shows the information of patents that comes from this class. 


\begin{tabular}{|l|l|l|}
\hline Team \# & \multicolumn{1}{|c|}{ Application Name } & Application Number \\
\hline Team 1 & A Solar Powered Hover Craft & $\mathbf{1 0 - 2 0 0 6 - 0 0 4 7 8 8 0}$ \\
\hline Team 1 & Solar Powered Balloon Toy to Propose Her & $\mathbf{1 0 - 2 0 0 6 - 0 0 4 5 5 1 4}$ \\
\hline Team 2 & An Auto Watering Machine with Timer & $\mathbf{1 0 - 2 0 0 6 - 0 0 4 6 3 0 8}$ \\
\hline Team 3 & A Bicycle with built-in a Fue1 Cell Powered Charger & $\mathbf{1 0 - 2 0 0 6 - 0 0 4 6 0 8 9}$ \\
\hline Team 4 & A Portable Capsule Shampoo & $\mathbf{1 0 - 2 0 0 6 - 0 0 4 6 3 1 2}$ \\
\hline Team 5 & A Reversely Holding Umbrella & $\mathbf{1 0 - 2 0 0 6 - 0 0 4 6 3 4 2}$ \\
\hline Team 6 & A Solar Powered Airship & $\mathbf{1 0 - 2 0 0 6 - 0 0 4 7 3 0 7}$ \\
\hline Team 7 & A Nude Coffee Mix Bar & $\mathbf{1 0 - 2 0 0 6 - 0 0 4 6 8 0 5}$ \\
\hline Team 8 & A Clothes Rack with Drying & $\mathbf{1 0 - 2 0 0 6 - 0 0 4 6 6 4 3}$ \\
\hline Team 9 & A Ruler with a Sliding Pen and a Spot Removal Machine & $\mathbf{1 0 - 2 0 0 6 - 0 0 4 6 3 5 2}$ \\
\hline Team 10 & A portable stain remover using a vacuum pump & $\mathbf{1 0 - 2 0 0 6 - 0 0 4 5 6 9 9}$ \\
\hline
\end{tabular}

Table4. Information of Patents

\section{Conclusions}

In Creative Design Class, forty-two students applied for patents successfully. Briefly, Students followed four steps of this class. In the first step, all students understood their type of preference through MBTI (Myers-Briggs Type Indicator) test. They also could take their grade of creativity through TTCT (Torrance Tests of Creative Thinking) test. The second step, students studied lectures related with improvement of creativity such as engineering design, technical administration, patent, product design, optimization and TRIZ by using method knowledge expansions in improvement of creativity. In third step, students made products with creative invention. For the last step, students finally applied for patents. In this class, students could get realistic experiences and upgrade their creativity as applying for patents.

\section{References}

1. Myers-Briggs Type Indicator, Wikipedia, the free encyclopedia, from http://en.wikipedia.org/wiki/Myers-Briggs_Type_Indicator

2. Torrance Tests of Creative Thinking, Mind Path Consulting Services, from http://www.mind-path.com/index_files/Page388.htm

3. United States Patent and Trademark Office, from http://www.uspto.gov/ 